\title{
Chandra observations of the globular cluster M 54
}

\author{
G. Ramsay and K. Wu
}

\author{
Mullard Space Science Laboratory, University College London, Holmbury St. Mary, Dorking, Surrey, RH5 6NT, UK \\ e-mail: gtbr@mssl.ucl.ac.uk
}

Received 19 July 2005 / Accepted 8 October 2005

\section{ABSTRACT}

We have carried out a Chandra observation of the globular cluster M 54. We detected 7 sources located within the half-mass radius of M 54, at a flux limit of $1.5 \times 10^{-15} \mathrm{erg} \mathrm{s}^{-1} \mathrm{~cm}^{-2}$ in the $0.3-8 \mathrm{keV}$ energy band. The spatial distribution and the colour/spectral properties of the 7 sources suggest that they are likely to be cataclysmic variables or LMXBs in the globular cluster. M 54 shows the largest number of X-ray sources with luminosities greater than $10^{32} \mathrm{erg} \mathrm{s}^{-1}$ compared to other globular clusters observed using Chandra and XMM-Newton. We searched for a correlation between the number of sources above this luminosity level with globular cluster parameters. We found evidence that the number of sources peaks at a King concentration parameter $c \sim 1.7-1.9$, with globular clusters which are core-collapsed or have low-c values having a smaller number of sources. We speculate on possible reasons for this.

Key words. Galaxy: globular clusters: individual: M 54 - X-rays: binaries - stars: binaries: general - Galaxy: globular clusters: general

\section{Introduction}

M 54 is a globular cluster located in the central region of the Sagittarius (Sgr) dwarf spheroidal galaxy (Ibata et al. 1994), which is at a distance of $27.4 \mathrm{kpc}$ (Layden \& Sarajedini 2000) and is the second closest satellite to the Milky Way. The host galaxy extends over at least $22^{\circ} \times 8^{\circ}$ in the sky, and is located on the far side of the Milky Way from the Sun (Ibata et al. 1994, 1995a). Some theories suggest that M 54 is the original nucleus of Sgr, but an alternative view is that the globular was actually captured (Monaco et al. 2003). In addition to M 54, several other globular clusters, Arp 2, Ter 7 and Ter 8, are also associated with the galaxy (Ibata et al. 1995b). Sgr is now being disrupted by the Milky Way, and these globulars might be the last remaining globular members that are still "attached" to Sgr.

Observations show that there is an excess of X-ray sources in Galactic globulars. It has been argued that processes in dense stellar environments (e.g. tidal capture and three-body interaction) are crucial in the formation and evolution of globular X-ray sources (e.g. Verbunt \& Hut 1987; Johnston \& Verbunt 1996; Pooley et al. 2003).

In 2003 we carried out a Chandra observation of the Sgr galaxy and the globular cluster M 54 to search for X-ray sources. Here we report our findings and present a brief comparison of the number of sources in M 54 with those in other Galactic globulars observed using Chandra.

\section{Observations and data analysis}

M 54 was observed using Chandra and the ACIS-I for $30 \mathrm{ks}$ on 2003 Sep. 3. The pointing was centered on $\alpha_{2000}=18^{\mathrm{h}} 55^{\mathrm{m}} 3.0^{\mathrm{s}}$, $\delta_{2000}=-30^{\circ} 28^{\prime} 59^{\prime \prime}:$ this was offset from the center of the globular cluster M 54 by $-12^{\prime \prime}$ in declination. The total field of view was $16.9^{\prime} \times 16.9^{\prime}$. Front illuminated CCDs $0-3$ were used. The read mode was configured in TIMING mode; the data-mode was VERY FAINT. During the observation the solar particle background was very low.

We used the primary data products as supplied by the Chandra Data Archive Operations. A source search was performed in the $0.3-8.0 \mathrm{keV}$ energy range using wavedetect (part of the CIAO v3.0 suite of software). This was done in conjunction with an exposure map using a mean photon energy of $1.5 \mathrm{keV}$. We rejected all "sources" with "significance" (as defined in the wavedetect routine) levels smaller than 3 . The resulting source list was then compared by-eye with the image in that band: "sources" which did not appear point-like were removed from the source list.

Seven sources were detected in the proximity of M 54 (Fig. 1) - all of them are located well within the cluster halfmass radius of 29.4" (Harris 1996). Source 2 could be a blend of two sources. Another 80 sources were detected elsewhere in the field - the "field" sources (Ramsay \& Wu, in prep.).

The positions and the photon counts of the M 54 sources are shown in Table 1. Based on the spectral analysis described in Sect. 4, we estimate that the flux sensitivity limits in the $0.3-8 \mathrm{keV}$ band is $1.5 \times 10^{-15} \mathrm{erg} \mathrm{s}^{-1} \mathrm{~cm}^{-2}$ (observed), $2.0 \times 10^{-15} \mathrm{erg} \mathrm{s}^{-1} \mathrm{~cm}^{-2}$ (unabsorbed) under the assumption that the sources have a power-law spectrum of photon index $\Gamma=1.7$ and a line-of-sight absorption column density of $1.2 \times 10^{21} \mathrm{~cm}^{-2}$. If we adopt a distance of $27.4 \mathrm{kpc}$ 
Table 1. The sources associated with the globular cluster M 54. The significance levels were derived using wavedetect in CIAO. The total energy band used was $0.3-8 \mathrm{keV}$. Soft counts refer to the $0.3-1 \mathrm{keV}$ band (S), medium counts the $1-2 \mathrm{keV}$ band (M) and hard counts the 2-8 keV band $(\mathrm{H})$, following the convention of Prestwich et al. (2003). ${ }^{*}$ In determining the X-ray luminosities we adopted a distance of $27.4 \mathrm{kpc}$ and made a count to flux conversion based on spectral fitting (see the description in Sect. 4).

\begin{tabular}{cccrrrrrr}
\hline \hline Id & RA & Dec & $\begin{array}{r}\text { Cts } \\
\text { (total) }\end{array}$ & Sig & $\begin{array}{r}\text { Cts } \\
\text { (soft })\end{array}$ & $\begin{array}{r}\text { Cts } \\
\text { (medium) }\end{array}$ & $\begin{array}{r}\text { Cts } \\
\text { (hard) }\end{array}$ & $\begin{array}{r}L_{\mathrm{x}}\left(10^{33} \mathrm{erg} \mathrm{s}^{-1}\right)^{*} \\
(0.5-2.5 \mathrm{keV})\end{array}$ \\
\hline 1 & 185502.96 & -302845.0 & 93.1 & 33.3 & 36.0 & 58.0 & 5.6 & 1.2 \\
2 & 185503.48 & -302847.4 & 57.0 & 17.5 & 12.4 & 25.7 & - & 0.72 \\
3 & 185503.89 & -302838.0 & 24.1 & 10.0 & 9.7 & 14.5 & - & 0.30 \\
4 & 185502.45 & -302857.5 & 18.0 & 7.8 & - & 7.8 & 9.6 & 0.23 \\
5 & 185503.65 & -302841.1 & 12.3 & 6.1 & - & - & 14.2 & 0.16 \\
6 & 185502.76 & -302853.0 & 10.6 & 5.5 & 3.7 & 8.5 & - & 0.13 \\
7 & 185502.66 & -302841.5 & 8.1 & 3.6 & - & - & 7.6 & 0.10 \\
\hline
\end{tabular}

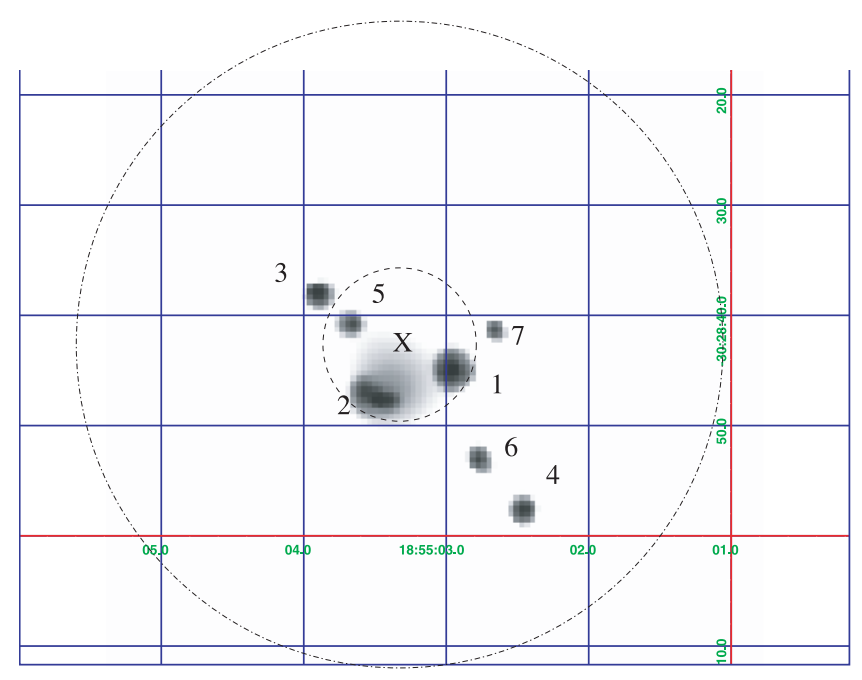

Fig. 1. The Chandra ACIS-I image showing the 7 sources in the globular cluster M 54. The image has been smoothed using the CIAO task csmooth. The cluster center (derived from contour plots of the Digital Sky Survey image of M 54) is marked by an " $X$ ". The core radius $\left(6.6^{\prime \prime}\right)$ and the half-mass radius $\left(29.4^{\prime \prime}\right)$ are denoted by concentric circles (values taken from Harris 1996).

(Layden \& Sarajedini 2000), then the lower limit for the unabsorbed bolometric X-ray luminosity is $1.5 \times 10^{32} \mathrm{erg} \mathrm{s}^{-1}$.

We determined the count rates for the sources in 3 energy bands, $0.3-1 \mathrm{keV}$ ("soft" $\equiv \mathrm{S}$ ), $1-2 \mathrm{keV}$ ("medium" $\equiv$ $\mathrm{M})$ and $2-8 \mathrm{keV}$ ("hard" $\equiv \mathrm{H}$ ), following the convention used by Prestwich et al. (2003), Soria \& Wu (2003) and Swartz et al. (2004) for the analysis of properties of X-ray sources in nearby spiral galaxies. The counts were determined using wavedetect and appropriate exposure maps. The count rates for the sources in the three bands are shown in Table 1. These 3 bands are used to derive the soft and hard X-ray colours.

For an assumed distance of $27.4 \mathrm{kpc}$ the brightest source in the M 54 field has an X-ray unabsorbed luminosity of $\sim 2 \times$ $10^{33} \mathrm{erg} \mathrm{s}^{-1}$ (in the $0.1-10 \mathrm{keV}$ energy band). This value is similar to those sources detected in the globular clusters 47 Tuc, NGC 6397, NGC 6440 and NGC 6752. Using the method of Wheatland (2004) we determined the slope of the source luminosity function to be $0.90 \pm 0.34$ using a lower cut-off of
8 counts. The slope of the luminosity function of the M 54 sources is steeper than those of the 4 globulars mentioned above (cf. Pooley et al. 2002b).

If the 80 field sources are a mixture of foreground and background sources, then we expect 0.2 field sources within the cluster half mass radius, or 0.01 within the core radius. This is similar to the predicted number of background AGN sources: the results of Rosati et al. (2002) predict 0.3 and 0.01 AGN within the cluster half mass and core radius respectively in the $2-10 \mathrm{keV}$ energy band. Further, if we remove all the sources detected in the whole field and sum up the remaining number of photons, we find that we expect 3.9 photons within an area equivalent to the cluster core radius or 0.029 photons per square arcsec. We conclude that the $7 \mathrm{X}$-ray sources are physically associated with the globular cluster.

\section{X-ray colours}

We derived the soft and hard colours $(\xi \equiv(\mathrm{M}-\mathrm{S}) /(\mathrm{H}+\mathrm{M}+\mathrm{S})$ and $\eta \equiv(\mathrm{H}-\mathrm{M}) /(\mathrm{H}+\mathrm{M}+\mathrm{S})$ respectively) for the sources following the convention used by Prestwich et al. (2003), Soria $\& \mathrm{Wu}$ (2003) and Swartz et al. (2004). Figure 2 shows the M 54 sources in the colour-colour plane.

We compared the colours of the sources with 3 spectral models: a blackbody, a power-law and an optically thin thermal plasma. In each model, we considered absorption column densities ranging from the Galactic hydrogen absorption density to the edge of the Galaxy $\left(1.2 \times 10^{21} \mathrm{~cm}^{-2}\right)$ to a column density of $N_{\mathrm{H}}=10^{24} \mathrm{~cm}^{-2}$. For the blackbody model we considered a temperature $k T_{\mathrm{bb}}=100 \mathrm{eV}$, appropriate for supersoft sources, and $k T_{\mathrm{bb}}=0.5$ and $1 \mathrm{keV}$, similar to the inner accretion-temperatures of X-ray binaries. For the power-law model we considered photon indices $\Gamma=1.4$ and 1.7, typical for X-ray binaries in the hard X-ray spectral state. The optically thin thermal plasma model we considered $\left(k T_{\text {th }}=10 \mathrm{keV}\right)$, is the temperature typical of the accretion shocks in magnetic CVs (mCVs). We also considered two lower temperatures $(0.5$ and $1.0 \mathrm{keV})$ for hot plasmas in supernova remnants and coronal stars. As an example, we show the colour-colour tracks of the blackbody models in Fig. 3. The other models follow similar colour tracks. 


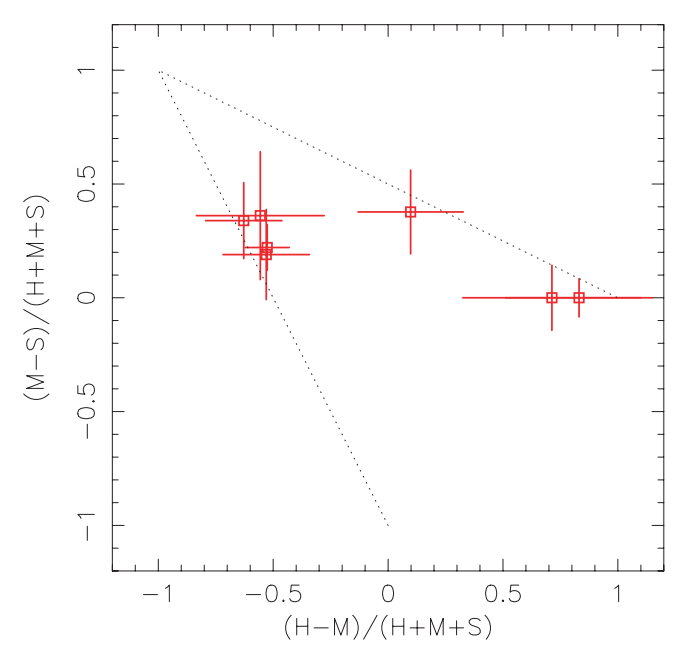

Fig. 2. The X-ray colour-colour diagrams of the sources in the globular cluster M 54. The error bars represent the uncertainties due to Poisson statistics of photon counts in the $\mathrm{S}(0.3-1 \mathrm{keV}), \mathrm{M}(1-2 \mathrm{keV})$ and $\mathrm{H}(2-8 \mathrm{keV})$ bands. The dotted lines enclose the possible colour-colour tracks for the models outlined in the text. (The abrupt change in the colour-colour track at $-1,1$ is due to the relatively coarse sampling of the line of sight absorption.)

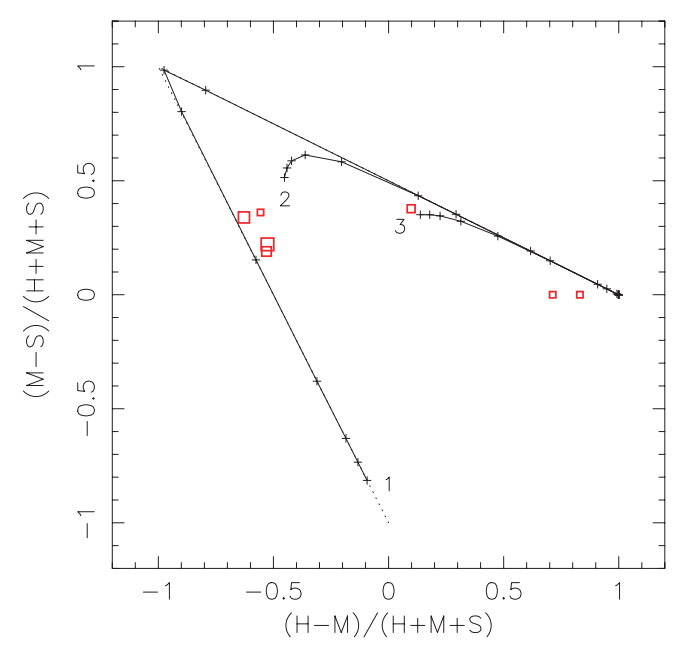

Fig. 3. The colours of the M 54 sources in comparison with tracks for the blackbody model with different temperatures and absorption column densities. The size of the symbol is proportional to the number of counts in the $0.3-8 \mathrm{keV}$ band. Tracks 1,2 and 3 correspond to the temperatures $k T_{\mathrm{bb}}=0.1,0.5$ and $1.0 \mathrm{keV}$ respectively. The absorption column density $n_{\mathrm{H}}$ starts at $1.2 \times 10^{21} \mathrm{~cm}^{-2}$ (the column density to the edge of the Galaxy in the Sgr direction), then steps through $5 \times$ $10^{21}, 1 \times 10^{22} \mathrm{~cm}^{-2}$ etc. For very high absorption column densities, the three colour tracks converge to $(1,0)$ in the colour-colour plane.

All the X-rays sources show colours with $-0.2<$ $(\mathrm{M}-\mathrm{S}) /(\mathrm{H}+\mathrm{M}+\mathrm{S})$. Soria \& Wu (2003) show that this region in the colour-colour diagram is populated by a collection of objects which may have a blackbody-like spectrum of $k T_{\text {in }} \sim$ $1 \mathrm{keV}$; an absorbed power-law spectrum with $\Gamma \sim 1.4-1.7$; or a spectrum of a hot $\left(k T_{\text {th }} \sim 10 \mathrm{keV}\right)$ optically thin thermal plasma. Objects such as accreting X-ray binaries and cataclysmic variables show such spectra.

\section{Luminosity estimates}

Estimating the luminosity of faint X-ray sources is not straightforward. However, we extracted a single spectrum from the 3 brightest soft M 54 sources and a single spectrum from a field source which was hard and relatively bright. We then fitted these spectra using a soft blackbody model and a blackbody plus power law model respectively (in addition to a neutral absorption model with absorption $1.2 \times 10^{21} \mathrm{~cm}^{-2}$ ). We then determined a mean counts to flux conversion; $1 \mathrm{ct}$ gives an unabsorbed flux of $1.4 \times 10^{-16} \mathrm{erg} \mathrm{s}^{-1} \mathrm{~cm}^{-2}$ in the $0.5-2.5 \mathrm{keV}$ energy band. Applying this mean conversion, instead of using either the soft or hard spectral model, gives an uncertainty of $30 \%$ in the luminosity. Extrapolating the spectra to an energy band of $0.1-10 \mathrm{keV}$ increases the inferred unabsorbed luminosity by a factor of 1.3 (for soft sources) and 4.5 (for hard sources).

Based on these conversions, we estimated the luminosity of the M 54 sources assuming a distance of $27.4 \mathrm{kpc}$. The sources have luminosities in the range $\sim 0.1-1.2 \times 10^{33} \mathrm{erg} \mathrm{s}^{-1}$ in the $0.5-2.5 \mathrm{keV}$ band. The luminosities of the sources are tabulated in Table 1.

The luminosity estimates suggest that the M 54 sources are unlikely to be main sequence stars, young stellar objects, RS CVn or Algol stars, which generally have X-ray luminosities less than $10^{32} \mathrm{erg} \mathrm{s}^{-1}$ (see Table 1 of Muno et al. (2003) and references therein). The source luminosities are consistent with those of mCVs, which have luminosities $\sim 10^{31-33} \mathrm{erg} \mathrm{s}^{-1}$. They are also in the range allowed for low mass X-ray binaries (LMXBs) and high mass X-ray binaries (HMXBs), which contain an accreting neutron star or black hole which are in quiescent accretion states. They are unlikely to be HMXBs since the stars in globular clusters are old. We suggest that the M 54 sources are most probably CVs or LMXBs.

\section{Comparison with other globular cluster sources}

A number of globular clusters have been observed using Chandra and XMM-Newton (e.g. M 80, see Heinke et al. 2003b). These observations show that the number of X-ray sources scales with the encounter rate (Pooley et al. 2003). Moreover, the source luminosities suggest that most of these systems are CVs, which are formed via a close encounter between a white dwarf and a low-mass star or a binary (see e.g. Verbunt 2004).

We have identified 7 sources within the half-mass radius of M 54. All 7 sources have inferred luminosities greater than $\sim 10^{32} \mathrm{erg} \mathrm{s}^{-1}$ in the $0.5-2.5 \mathrm{keV}$ band. We compare the number of sources in M 54 with those of other globular clusters in the same energy band. For those systems where it was possible to make this comparison we show in Table 2 the number of sources in each cluster which have luminosities greater than $10^{32} \mathrm{erg} \mathrm{s}^{-1}(0.5-2.5 \mathrm{keV}$ band). Compared to the globular clusters noted in Table 2, M 54 has more sources with luminosities above $1 \times 10^{32} \mathrm{erg} \mathrm{s}^{-1}$.

M 54 is one of the most optically luminous globular clusters associated with the Milky Way or its satellites (Harris 1996). We searched for a correlation between the number of X-ray 
Table 2. The number of X-ray sources in different globular clusters which have luminosities greater than $1 \times 10^{32} \mathrm{erg} \mathrm{s}^{-1}$ (observed) or $2.5 \times 10^{32} \mathrm{erg} \mathrm{s}^{-1}$ (unabsorbed), depending on what is quoted in the literature. References: (1) Pooley et al. (2002b); (2) Heinke et al. (2003a); (3) Grindlay et al. (2001a); (4) Heinke et al. (2003b); (5) Becker et al. (2003); (6) Pooley et al. (2002a); (7) Bassa et al. (2004); (8) Grindlay et al. (2001b); (9) Webb et al. (2002); (10) Gendre et al. (2003).

\begin{tabular}{lrr}
\hline \hline Cluster & Sources & Reference \\
\hline NGC 6440 & 6 & $(1)$ \\
Terzan 5 & 5 & $(2)$ \\
47 Tuc & 2 & $(3)$ \\
M 80 & 5 & $(4)$ \\
M 28 & 5 & $(5)$ \\
NGC 6752 & 1 & $(6)$ \\
M 4 & 0 & $(7)$ \\
NGC 6397 & 1 & $(8)$ \\
M 22 & 1 & $(9)$ \\
$\omega$ Cen & 4 & $(10)$ \\
\hline
\end{tabular}

sources above $1 \times 10^{32} \mathrm{erg} \mathrm{s}^{-1}$ and the system parameters of the host globular cluster. We considered only global parameters measurable by observations or derived directly and explicitly from observations. We ignored local parameters or derived parameters which are based on models which invoke implicit assumptions. We found no convincing evidence for a correlation between the source number and the central surface brightness, the core radius, the half-mass radius, the tidal radius, the relaxation time, the metallicity or the distance to the Galactic center (see Fig. 4).

However, when we plotted the source number against the King (1966) concentration ( $c=\log \left[r_{\mathrm{t}} / r_{\mathrm{c}}\right]$, where $r_{\mathrm{t}}$ is the tidal radius and $r_{\mathrm{c}}$ is the core radius), we noticed that the source number peaks at $c \approx 1.7-1.9$. Core-collapsed globular clusters and low- $c$ globular clusters appear to have fewer sources brighter than $\sim 10^{32} \mathrm{erg} \mathrm{s}^{-1}$ (Fig. 4). A simple KS test (against a uniform distribution with respect to $c$ ) yielded a $D$-statistic of 0.356 . The critical $D$ value at the significant level $\alpha=0.01$ is 0.268 . Thus, the peak at $c \approx 1.8$ is unlikely to be due to statistical fluctuations.

If we accept that the peak is genuine, this may indicate that too high a concentration of stars in the core of a globular cluster reduces the number of binary X-ray sources. This could be due to the formation rate of binaries being less than the dissociation rate; another possibility could be that a substantial fraction of binaries have been ejected from the high-density core of the collapsed globular clusters (R. Taam, private communication). More globular clusters (especially those with large and small $c$ ) are needed to fully confirm this result.

\section{Summary}

We have undertaken an X-ray survey of the globular cluster M 54. We search down to a flux limit of $1.5 \times$ $10^{-15} \mathrm{erg} \mathrm{s}^{-1} \mathrm{~cm}^{-2}$ in the $0.3-8 \mathrm{keV}$ band. A total of 7 sources were identified as located within half-mass radius of M 54 .
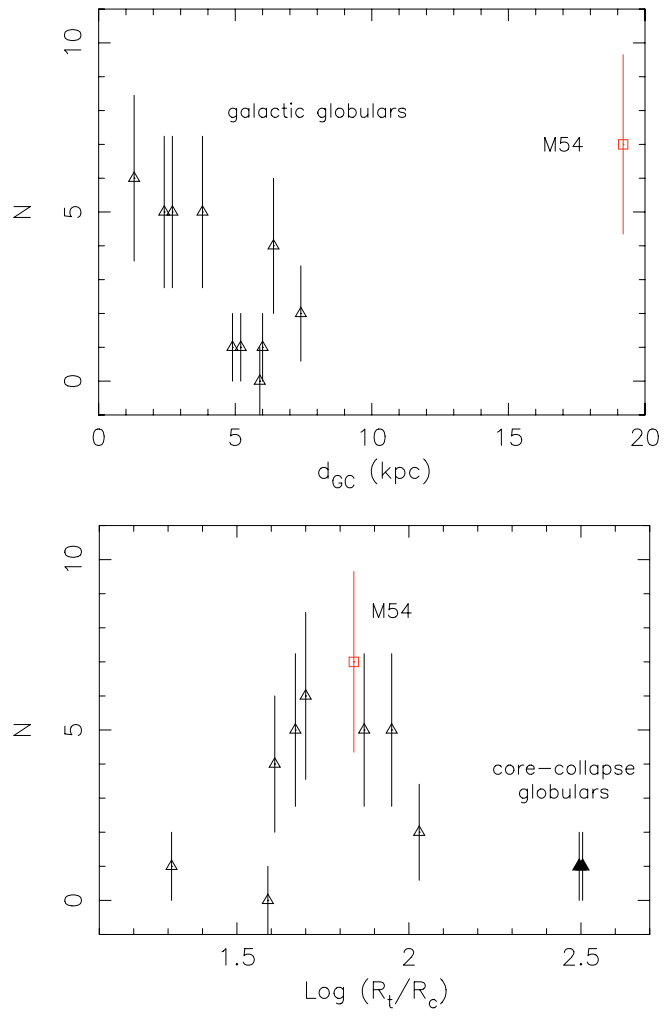

Fig. 4. Top panel: the number of sources in globular clusters with $L_{\mathrm{X}}>1 \times 10^{32} \mathrm{erg} \mathrm{s}^{-1}(0.5-2.5 \mathrm{keV})$ as a function of distance of the cluster to the Galactic center. Bottom panel: the number of sources in globular clusters as a function of the King (1966) concentration. The core-collapsed globular clusters, which are represented by filled triangles, are assumed to have $c \equiv \log \left[r_{\mathrm{t}} / r_{\mathrm{c}}\right]=2.5$.

The over-density of sources within the half-mass radius of M 54 in comparison with the field sources indicates that the 7 sources belong to the globular cluster and are not likely to be foreground stars or background AGN.

The colours and the inferred luminosities suggest that the M 54 sources are accreting systems, likely to be magnetic CVs or LMXBs. By comparison with other globular clusters observed by Chandra and XMM-Newton, M 54 is among those with most "luminous" X-ray sources (with $L_{\mathrm{x}}>10^{32} \mathrm{erg} \mathrm{s}^{-1}$ ). There appears to be a range of central concentration $(c \approx$ 1.7-1.9) where the globular clusters have a significant number of bright sources.

Acknowledgements. We thank Michael Wheatland for correspondence and discussion regarding Bayesian statistics. We thank Allyn Tennant for correspondence in source analysis and Ron Taam for discussion in X-ray sources in globular clusters. We also thank Doug Swartz and Kajal Ghosh for discussions and the referee for useful comments.

\section{References}

Bassa, C., Pooley, D., Homer, L., et al. 2004, ApJ, 609, 755

Becker, W., Swartz, D. A., Pavlov, G. G., et al. 2003, ApJ, 594, 798

Gendre, B., Barret, D., \& Webb, N. A. 2003, A\&A, 400, 521

Grindlay, J. E., Heinke, C., Edmonds, P. D., \& Murray, S. S. 2001a, Science, 292, 2290 
Grindlay, J. E., Heinke, C., Edmonds, P. D., Murray, S. S., \& Cool, A. M. 2001b, ApJ, 563, L53

Harris, W. E. 1996, AJ, 112, 1487

Heinke, C. O., Edmonds, P. D., Grindlay, J. E., et al. 2003a, ApJ, 590, 809

Heinke, C. O., Grindlay, J. E., Edmonds, P. D., et al. 2003b, ApJ, 598, 516

Ibata, R. A., Gilmore, G., \& Irwin, M. J. 1994, Nature, 370, 194

Ibata, R. A., Gilmore, G., \& Irwin, M. J. 1995a, MNRAS, 277, 781

Ibata, R. A., Gilmore, G., Irwin, M. J., et al. 1995b, MNRAS, 277, 781

Johnston, H. M., \& Verbunt, F. 1996, A\&A, 312, 80

King, I. R. 1966, AJ, 71, 64

Layden, A. C., \& Sarajedini, A. 2000, AJ, 119, 1760

Monaco, L., Bellazzini, M., Ferraro, F. R., \& Pancino, E. 2003, in Proc. Satellites and Tidal Streams, ed. F. Prada, D. Martinez-Delgado, \& T. Mahoney, in press

[arXiv: astro-ph/0309044]
Muno, M. P., Baganoff, F. K., Bautz, M. W., et al. 2003, ApJ, 589, 225 Pooley, D., Lewin, W. H. G., Homer, L., et al. 2002a, ApJ, 569, 405 Pooley, D., Lewin, W. H. G., Verbunt, F., et al. 2002b, ApJ, 573, 184 Pooley, D., Lewin, W. H. G., Anderson, S. F., et al. 2003, ApJ, 591, L131

Prestwich, A., Irwin, J. A., Kilgard, R. E., et al. 2003, ApJ, 595, 719 Rosati, P., Tozzi, P., Giacconi, R., et al. 2002, ApJ, 566, 667

Soria, R., \& Wu, K. 2003, A\&A, 410, 56

Swartz, D. A., Ghosh, K. K., Tennant, A. F., \& Wu, K. 2004, ApJS, 154,519

Verbunt, F. 2004, in Compact Stellar X-ray Sources, ed. W. H. G. Lewin, \& M. van der Klis (Cambridge University Press), to appear [arXiv: astro-ph/0404136]

Verbunt, F., \& Hut, P. 1987, in The Origin and Evolution of Neutron Stars, ed. D. J. Helfand, \& J. H. Huang (Dordrecht: Reidel), IAU Symp., 125, 187

Webb, N. A., Gendre, B., \& Barret, D. 2002, A\&A, 381, 481

Wheatland, M. S. 2004, ApJ, 609, 1134 